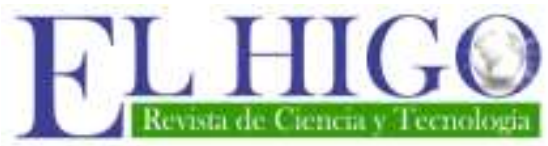

Vol. 07. pp. 02-10/Diciembre 2017

\title{
COMPARACIÓN MÉTODO HARDY CROSS Y SOFWARE EPANET EN DISEÑO DE REDES DE AGUA POTABLE
}

\section{METHOD HARDY CROSS AND SOFTWARE EPANET IN DRINKING WATER SYSTEMS DESIGN COMPARISON}

\author{
Yeraldo Andrés Rodríguez Molina ${ }^{1}$ \\ Henry Eduardo Loaísiga ${ }^{2}$
}

(recibido/received: 25-Julio-2017; aceptado/accepted: 28-Octubre-2017)

RESUMEN: Los problemas de hidráulica de tuberías (por ejemplo, proyectos de sistemas de abastecimiento de agua potable) históricamente se han venido resolviendo por medio del método "empírico" de Hazen Williams debido a su naturaleza explícita. Sin embargo, dicho método tiene restricciones para su aplicabilidad, las que muchas veces no se tienen en cuenta en el proceso de diseño. Razón por la cual es importante retomar la metodología con fundamento en la física clásica como es el caso del método de Darcy - Weisbach que no posee limitación alguna. Con ayuda de las computadoras este método deja de ser tan engorroso como en sus inicios (1936). Para la presente investigación se emplearon los métodos de cálculo de pérdidas de carga por fricción en tuberías de Darcy - Weisbach en conjunto con la ecuación de Colebrook - White utilizando como valor de referencia la ecuación de Swamee - Jain en un proceso de análisis numérico iterativo de punto fijo para calcular el factor de fricción de Darcy en tuberías. Con lo cual fue posible implementar el método de "Hardy Cross con Corrección de Caudales" en la red de distribución y, posteriormente, observar las variaciones con respecto al software EPANET. Se demostró que el uso de ambos métodos genera resultados por debajo de $1 \%$ de variabilidad, lo cual, evidencia que ambas metodologías son congruentes. La convergencia presenta una considerable sensibilidad a las variaciones en la precisión decimal.

PALABRAS CLAVE: Agua potable; hidráulica de tuberías; Darcy - Weisbach.

\footnotetext{
${ }^{1}$ Ingeniero Civil - Docente Horario de UNI RUACS, Estelí-Nicaragua.ing.yeraldorodriguez@gmail.com.

2 Ingeniero Hidrotécnico. Director Universidad Nacional de Ingeniería Sede Regional del Norte (UNI RUACS) EstelíNicaragua,henry.loaisiga@uni.edu.ni.
} 
ABSTRACT: The problems of pipe hydraulics (for example: Potable Water Supply Systems Projects) historically have come to solve by Hazen Williams' "empirical" method due to its explicit nature. However, this method has restrictions for its application, which often do not take into account in the design process. Reason why it is important to return to the methodology based on classical physics as the case of the method of Darcy - Weisbach wich don't have any limits. With the help of computers this method is no longer complicated as in its beginnings (1936). For current research were use power loss by friction in pipes calculation method's IDarcy - Weisbach 's in conjunction with the Colebrook - White equation using the Swamee - Jain equation as a reference value in a fixed point iterative numerical analysis process to calculate Darcy' $s$ friction factor in pipes. With wich was posible implemented the method of "Hardy Cross with Correction of Flows" in the distribution network and, later, observe the variations in respect to the EPANET software. It was demonstrated that the use of both methods generates results below $1 \%$ of variability, which shows that both methodologies are congruent. Convergence exhibits a considerable sensitivity to changes in decimal precision.

KEYWORDS: Drinking water; pipeline hydraulics; Darcy - Weisbach.

\section{INTRODUCCIÓN}

El profesor Hardy Cross desarrolló su método inicialmente para dar solución al problema de vigas continuas en análisis estructural; sin embargo, rápidamente adaptó su metodología para la solución en hidráulica de las redes cerradas, propias de los sistemas de abastecimiento de agua potable en el año 1936.

La presente investigación se realizó porque es necesario documentar la importancia de retomar el Método de Cross a través del cálculo de pérdidas por fricción con Darcy - Weisbach, la cual ha ido quedando en desuso por la complejidad que involucra la obtención de los factores de fricción en la ecuación de Colebrook - White. Tal como lo expone (Saldarriaga, 2007): "El hecho de que la anterior ecuación sea no explícita para el factor de fricción $f$ implica que la solución de problemas de tuberías debe incluir métodos numéricos iterativos, lo cual, antes de la popularización de los computadores, hacía que su uso fuera engorroso".

Los resultados reflejados en este artículo muestran un ejemplo práctico que involucra la aplicación del Método de Cross utilizando la ecuación de Darcy - Weisbach a través de hojas de cálculo y la posterior comparación con resultados en EPANET el cual utiliza el "Método del Gradiente" dentro de su algoritmo (método matricial desarrollado por Todini y Pilati en 1987).

\section{METODOLOGÍA}

El análisis comparativo entre ambas metodologías de diseño para redes de agua potable se inició con la realización de los "estudios previos" (entre los que se destacan el censo poblacional, topografía, entre otros). Luego se establecieron los "parámetros de diseño" según la normativa NTON 09-001-99 (INAA, 1999). Posteriormente, se llevó a cabo un "estudio de consumo" para 
idealizar la demanda concentrada en cada nodo partiendo de los parámetros establecidos anteriormente, la proyección poblacional geométrica, caudales de diseño y planos.

Partiendo de esta información se procedió a la "distribución interna de los caudales" en los tramos de la red que sería capaz de suministrar las demandas concentradas en los nodos, respetando en todo momento el "Principio de Conservación de Masa" y tomando como referencia la dirección del reloj como positiva para cada circuito de la red.

Después se creó una base de datos para registrar las características de la red (coordenadas, etiquetas para nodos y tramos, longitud, diámetro, rugosidad absoluta, coeficiente de pérdida menor global y la distribución interna de caudales).

Tomando esta base de datos se procedió al cálculo de pérdidas de carga por fricción. Para este caso, se eligió el método de Darcy - Weisbach en conjunto con Colebrook - White. Las razones por las cuales se toma esta decisión se deben a lo expuesto por Saldarriaga (2007) quién con mucho detalle explica las limitaciones y beneficios de ambas metodologías. En resumen, la ecuación físicamente basada es Darcy - Weisbach la cual no tiene limitación alguna; mientras, la ecuación de Hazen - Williams es de carácter empírico y presenta limitantes o restricciones ya que es aplicable única y exclusivamente para agua a temperatura normal, en diámetros iguales o mayores a $75 \mathrm{~mm}$ ( 3 pulgadas) y la velocidad debe ser inferior a $3 \mathrm{~m} / \mathrm{s}$.

$$
\begin{aligned}
& \text { Darcy - Weisbach } \quad h_{f}=f \frac{L}{D} \frac{V^{2}}{2 g} \quad \text { (Ec.1) } \\
& \text { Colebrook - White } \frac{1}{\sqrt{f}}=-2 \log _{10}\left(\frac{\varepsilon / D}{3.7}+\frac{2.51}{\operatorname{Re} \sqrt{f}}\right)
\end{aligned}
$$

Se eligió el método de análisis numérico "Iteración de Punto Fijo" documentado por los autores Chapra \& Canale (2007) para encontrar la raíz de la ecuación del factor de fricción Colebrook White ya que su programación es menos compleja comparada con el método de Newton. Posteriormente, se aplicó el "Método de Cross con Corrección de Caudales" asumiendo para la primera prueba iterativa la distribución interna de caudales iniciales. Los principios que se aplican son: "Conservación de Masa y Conservación de Energía".

$$
\begin{aligned}
& \text { Iteración de punto fijo } \quad x_{i+1}=f\left(x_{i}\right) \quad \text { (Ec.3) } \\
& \text { Swamee - Jain (valor semilla) } f=\frac{0.25}{\left[\log \left(\frac{\epsilon / D}{3.71}+\frac{5.74}{R e^{0.9}}\right)\right]^{2}} \\
& \text { Pérdida de carga total } \quad H_{f}=\frac{8 Q^{2}}{\pi^{2} g D_{i}^{4}}\left(f_{i} \frac{L_{i}}{D_{i}}+\sum_{i=1}^{n} K_{m_{i}}\right)
\end{aligned}
$$


El primer principio se aplica en los nodos y se logra al proponer caudales iniciales para los tramos de tuberías que convergen a dichos nodos en base a las demandas concentradas y respetando que la sumatoria de los flujos entrantes y salientes en cada nodo debe ser igual a cero. El segundo principio se aplica a los circuitos y se cumple a través de iteraciones sucesivas hasta lograr que la sumatoria de pérdidas de carga en los circuitos tienda a cero, lo que provoca que las variaciones o correcciones del caudal también tiendan a cero, balanceando de esta forma la red de distribución.

Para la modelación hidráulica en EPANET primero se realizó el trazo geométrico de la red, luego se editaron las propiedades de los objetos que conforman el sistema. Para agilizar el trabajo, disminuir el esfuerzo y evitar errores en la digitalización de datos, se tomó la base de datos inicial y se procesó con ayuda de la hoja de cálculo EPA_EXCEL de la Universidad Técnica Particular de Loja para exportar a través de un archivo con extensión *.INP que puede ser importado con EPANET. Posteriormente, se determinaron las opciones de análisis hidráulico según lo explica (Rossman, 1997) en su manual de usuario, se ejecutó el programa para un análisis en régimen permanente (simulación estática) y finalmente se extrajeron los resultados para la comparación con el Método de Cross realizado con hojas de cálculo.

\section{RESULTADOS Y DISCUSIÓN}

Para aplicar esta comparación se hizo uso de una red cerrada, donde se llevó a cabo idealizando el circuito principal en tramos entre 200 y 300 m, luego se asignaron las etiquetas en nodos y tramos para una correcta identificación. Con ayuda del plano catastral se definieron las salidas de caudal haciendo una asignación nodo por nodo. El material propuesto fue PVC. Esta información se registró en una tabla de datos en conjunto con las demás características de los tramos de la red como punto de partida para el análisis, tal como se aprecia en la Tabla 1. Una de las observaciones que merecen la pena mencionar es el hecho de que los diámetros que deben ser utilizados para los diseños deben ser los diámetros internos de las tuberías y no los diámetros nominales. Según (Mott, 2006, pág. 160) así como

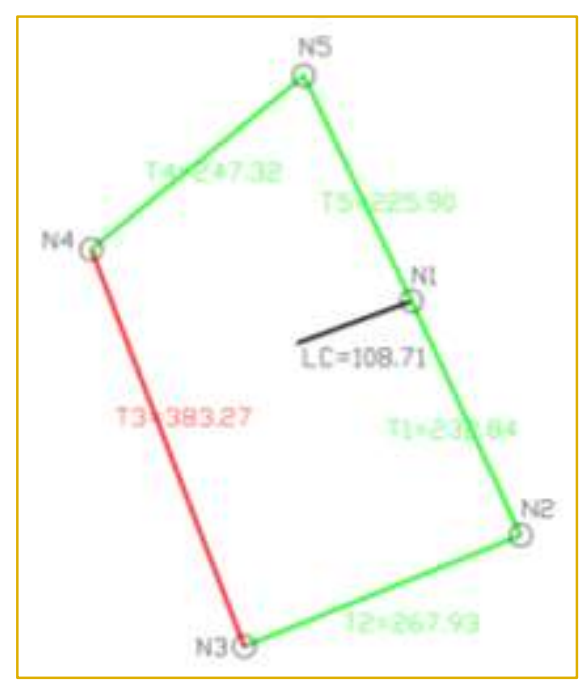
(Saldarriaga, 2007, pág. 87): "para efectos de diseño se deben emplear diámetros internos de las tuberías ya que los diámetros nominales sirven netamente para nombrar las tuberías, sin embargo, no expresan el valor real del diámetro interno en los cálculos". Para tubería PVC SDR 26 correspondiente a la norma ASTM D-2241, el diámetro nominal de 2" equivale a un diámetro interno de $55.70 \mathrm{~mm}$, asignando $50 \mathrm{~mm}$ el error cometido es $-10.23 \%$ : 
Tabla 1: Circuito principal

\begin{tabular}{|c|c|c|c|c|c|c|c|c|c|}
\hline \multirow{2}{*}{ Id } & \multirow{2}{*}{ Circuito } & \multicolumn{2}{|c|}{ Nodos } & \multirow{2}{*}{ Tramo } & \multirow{2}{*}{$\begin{array}{c}\text { Longitud } \\
\mathrm{M}\end{array}$} & \multirow{2}{*}{$\begin{array}{c}\text { Diámetro } \\
\mathrm{mm}\end{array}$} & \multirow{2}{*}{$\begin{array}{c}\text { Rugosidad } \\
\mathrm{mm}\end{array}$} & \multirow{2}{*}{$\Sigma K_{m}$} & \multirow{2}{*}{$\begin{array}{c}\mathbf{Q}_{\mathbf{i}}(\mathbf{R}+) \\
\mathrm{m}^{3} / \mathrm{s}\end{array}$} \\
\hline & & $\mathrm{N}_{\mathrm{i}}$ & $N_{f}$ & & & & & & \\
\hline 1 & \multirow{6}{*}{1} & N1 & N2 & $\mathrm{T} 1$ & 232.84 & 55.70 & 0.0015 & 0 & 1.44E-03 \\
\hline 2 & & N2 & N3 & T2 & 267.93 & 44.55 & 0.0015 & 0 & 6.86E-04 \\
\hline 3 & & N3 & N4 & T3 & 383.27 & 44.55 & 0.0015 & 0 & $-6.39 E-07$ \\
\hline 4 & & N4 & N5 & T4 & 247.32 & 44.55 & 0.0015 & 0 & $-8.82 E-04$ \\
\hline 5 & & N5 & N1 & T5 & 225.90 & 55.70 & 0.0015 & 0 & $-1.44 \mathrm{E}-03$ \\
\hline 6 & & N1 & & & & & & & \\
\hline
\end{tabular}

Fuente: Elaboración propia

La distribución de caudales iniciales $\left(Q_{i}\right)$ se calculó aplicando el Principio de Conservación de Masa en cada nodo tomando el sentido de las manecillas del reloj como positivo $(\mathrm{R}+)$.

Tabla 2: Cálculo del factor de fricción

\begin{tabular}{|c|c|c|c|c|c|}
\hline \multirow{2}{*}{ Id } & $\mathbf{Q}_{\text {prueba }}$ & \multirow{2}{*}{$\mathrm{Re}$} & \multirow{2}{*}{ f Swamee Jain } & \multirow{2}{*}{$f_{\text {prueba }}$} & \multirow{2}{*}{$f$} \\
\hline & $\mathrm{m}^{3} / \mathrm{s}$ & & & & \\
\hline 1 & 1.49E-03 & 38,920 & 0.02208 & 0.02219 & 0.02219 \\
\hline 2 & 7.34E-04 & 23,968 & 0.02477 & 0.02485 & 0.02485 \\
\hline 3 & 4.76E-05 & 1,552 & - & - & 0.04124 \\
\hline 4 & $-8.34 \mathrm{E}-04$ & 27,212 & 0.02403 & 0.02412 & 0.02412 \\
\hline 5 & $-1.39 E-03$ & 36,404 & 0.02242 & 0.02253 & 0.02253 \\
\hline 6 & & & & & \\
\hline
\end{tabular}

Fuente: Elaboración propia

Tabla 3. Método de Cross: cálculo de pérdidas por fricción y caudales corregidos

\begin{tabular}{|c|c|c|c|c|c|c|}
\hline I & $\mathbf{Q}_{\text {prueba }}$ & \multirow{2}{*}{$\mathbf{K}$} & $H_{f}$ & $\mathrm{H}_{\mathrm{f}} / \mathbf{Q}$ & $\Delta Q$ & $\mathbf{Q}_{\text {corregido }}$ \\
\hline & $\mathrm{m}^{3} / \mathrm{s}$ & & $\mathrm{m}$ & $\mathrm{s} / \mathrm{m}^{2}$ & $\mathrm{~m}^{3} / \mathrm{s}$ & $\mathrm{m}^{3} / \mathrm{s}$ \\
\hline 1 & 1.49E-03 & $796,354.09$ & 1.771 & $1,187.48$ & \multirow{6}{*}{0.00000} & 1.49E-03 \\
\hline 2 & 7.34E-04 & $3135,118.79$ & 1.691 & $2,302.64$ & & 7.34E-04 \\
\hline 3 & 4.76E-05 & $7442,038.58$ & 0.017 & 353.92 & & 4.76E-05 \\
\hline 4 & $-8.34 \mathrm{E}-04$ & $2808,455.25$ & -1.953 & $2,341.87$ & & $-8.34 \mathrm{E}-04$ \\
\hline 5 & $-1.39 E-03$ & $784,408.78$ & -1.526 & $1,094.06$ & & $-1.39 E-03$ \\
\hline 6 & & $\Sigma$ & 0.000 & $7,279.98$ & & \\
\hline
\end{tabular}

Fuente: Elaboración propia 
Los resultados se obtienen a través de la aplicación del "Método de Cross" el "Principio de Conservación de la Energía" entre el nodo de alimentación a la red y el nodo crítico, siguiendo la metodología presentada por Lanza (2012) para la determinación de la presión necesaria a la entrada de la red de distribución que asegure la presión mínima de 5.00 m.c.a en el punto crítico bajo el escenario de diseño de consumo máximo horario.

Tabla 4: Resultados según CROSS

\begin{tabular}{|c|c|c|c|c|c|c|}
\hline \multirow{2}{*}{ Tramo } & Caudal & Velocidad & Pérd. Unit. & \multirow{2}{*}{$\begin{array}{l}\text { Factor de } \\
\text { Fricción }\end{array}$} & \multirow{2}{*}{ Nodo } & Presión \\
\hline & Ips & $\mathrm{m} / \mathrm{s}$ & $\mathrm{m} / \mathrm{km}$ & & & $\mathrm{m}$ \\
\hline $\mathrm{T} 1$ & 1.49 & 0.61 & 7.60 & 0.02219 & N1 & 9.46 \\
\hline $\mathrm{T} 2$ & 0.73 & 0.47 & 6.31 & 0.02485 & N2 & 8.69 \\
\hline T3 & 0.05 & 0.03 & 0.04 & 0.04124 & ${ }^{* * *}$ N3 ${ }^{* * *}$ & ${ }^{* * *} 5.00^{* * *}$ \\
\hline $\mathrm{T} 4$ & 0.83 & 0.53 & 7.90 & 0.02412 & N4 & 5.98 \\
\hline T5 & 1.39 & 0.57 & 6.75 & 0.02253 & N5 & 10.93 \\
\hline
\end{tabular}

Fuente: Elaboración propia

Las velocidades son satisfactorias en cuatro tramos de la red y solo un tramo queda fuera de rango $(\mathrm{T} 3=0.03 \mathrm{~m} / \mathrm{s})$ según parámetro de diseño $(0.40 \leq \mathrm{V}(\mathrm{m} / \mathrm{s}) \leq 2.00)$. Las pérdidas unitarias presentan un comportamiento aceptable por debajo de $10 \mathrm{~m} / \mathrm{km}$ en todos los tramos. Manifestándose el nodo tres (N3) como punto crítico.

Tabla 5: Resultados según EPANET

\begin{tabular}{|c|c|c|c|c|c|c|}
\hline Tramo & $\begin{array}{c}\text { Caudal } \\
\text { Ips }\end{array}$ & $\begin{array}{c}\text { Velocidad } \\
\mathrm{m} / \mathrm{s}\end{array}$ & $\begin{array}{l}\text { Pérd. Unit. } \\
\text { m/km }\end{array}$ & Factor de Fricción & Nodo & $\begin{array}{c}\text { Presión } \\
\text { m }\end{array}$ \\
\hline $\mathrm{T} 1$ & 1.49 & 0.61 & 7.60 & 0.02219 & N1 & 9.46 \\
\hline $\mathrm{T} 2$ & 0.73 & 0.47 & 6.32 & 0.02490 & N2 & 8.69 \\
\hline T3 & 0.05 & 0.03 & 0.04 & 0.04219 & ${ }^{* * *} \mathrm{~N} 3{ }^{* \star *}$ & *** 5.00 *** \\
\hline $\mathrm{T} 4$ & 0.83 & 0.53 & 7.90 & 0.02415 & N4 & 5.98 \\
\hline T5 & 1.39 & 0.57 & 6.75 & 0.02254 & N5 & 10.94 \\
\hline
\end{tabular}

Fuente: Elaboración propia 
Tabla 6: Variaciones en resultados: CROSS - EPANET

\begin{tabular}{|c|c|c|c|c|c|c|}
\hline Tramo & $\begin{array}{c}\text { Caudal } \\
\text { Ips }\end{array}$ & $\begin{array}{c}\text { Velocidad } \\
\mathrm{m} / \mathrm{s}\end{array}$ & $\begin{array}{c}\text { Pérd. Unit. } \\
\text { m/km }\end{array}$ & Factor de Fricción & Nodo & $\begin{array}{c}\text { Presión } \\
\mathrm{m}\end{array}$ \\
\hline $\mathrm{T} 1$ & $0.00 \%$ & $0.00 \%$ & $0.06 \%$ & $0.00 \%$ & N1 & $-0.04 \%$ \\
\hline $\mathrm{T} 2$ & $0.01 \%$ & $0.01 \%$ & $-0.15 \%$ & $-0.20 \%$ & N2 & $-0.06 \%$ \\
\hline T3 & $0.13 \%$ & $0.13 \%$ & $-1.96 \%$ & $-2.25 \%$ & N3 & $-0.05 \%$ \\
\hline $\mathrm{T} 4$ & $-0.01 \%$ & $-0.01 \%$ & $-0.11 \%$ & $-0.12 \%$ & N4 & $-0.04 \%$ \\
\hline T5 & $0.00 \%$ & $0.00 \%$ & $0.02 \%$ & $-0.04 \%$ & N5 & $-0.04 \%$ \\
\hline
\end{tabular}

Fuente: Elaboración propia

\section{CONCLUSIONES}

Usar diámetros nominales en vez del diámetro interno real de las tuberías tiende a calcular pérdidas de carga por fricción mayores a las reales. El cálculo de la longitud de las tuberías por lo general se realiza con ayuda de las coordenadas de los levantamientos topográficos. Para lo cual, resulta más apegado a la realidad emplear la longitud euclídea 3D y no la 2D.

El análisis hidráulico se realizó con hojas de cálculo y modelación en EPANET dando como resultado variaciones por debajo del $1 \%$ (en su mayoría) entre ambas metodologías. La sensibilidad depende de las cifras decimales para la convergencia entre ambas metodologías de comprobación de diseño (Cross o EPANET). En esta investigación se configuraron las hojas de cálculo con formatos condicionales que identificaban la convergencia con una precisión de \pm $0.00001 \mathrm{~m} 3 / \mathrm{s}$ para el caudal y \pm 0.001 metros para las pérdidas de carga por fricción. Sin embargo, la ventaja de las hojas de cálculo es la versatilidad de poder modificar y mejorar la precisión, en cualquier momento si así se desea.

Es posible el uso de la ecuación de Swamee - Jain como valor semilla en el proceso iterativo de punto fijo para estimar con mayor exactitud el factor de fricción a través de una metodología de análisis numérico para la posterior incorporación en el cálculo de pérdida de carga con la ecuación de Darcy - Weisbach. Las ecuaciones racionales y físicamente basadas como es el caso de Darcy - Weisbach en conjunto con Colebrook - White para el cálculo de las pérdidas por fricción en tuberías no tienen limitación alguna; además, su formulación está basada en la física clásica, tal como lo expone y señala rotundamente (Saldarriaga, 2007).

Dado que el método de Hardy Cross realiza análisis para un escenario estático (estado permanente), es recomendable realizar análisis en período extendido en EPANET, con patrones de consumo para Nicaragua. De esta manera sería posible el estudio de coeficientes de regularización de consumo más apegados a la realidad en la comprobación de diseño tanto de redes de distribución como para el dimensionamiento de tanques de almacenamiento. 


\section{SIMBOLOGIA}

$\boldsymbol{h}_{\boldsymbol{f}}$ : Pérdida de carga por fricción en (m.c.a).

$f$ : Factor de fricción (adimensional).

$\boldsymbol{L}$ : Longitud en (m).

D: Diámetro interno de la tubería en $(\mathrm{m})$.

$V$ : Velocidad en $(\mathrm{m} / \mathrm{s})$.

$\boldsymbol{g}$ : Aceleración de la gravedad $\left(9.81 \mathrm{~m} / \mathrm{s}^{2}\right)$.

$\varepsilon$ : Rugosidad absoluta del material en $(\mathrm{m})$.

$\boldsymbol{R e}$ : Número de Reynolds (adimensional).

$\boldsymbol{H}_{f}$ : Pérdida de carga total en (m.c.a).

$Q$ : Caudal en $\left(\mathrm{m}^{3} / \mathrm{s}\right)$.

$\boldsymbol{K}_{\boldsymbol{m}}$ : Coeficiente de pérdida por accesorio (adimensional)

\section{REFERENCIAS}

Chapra, S., \& Canale, R. (2007). Métodos Numéricos para Ingenieros (Quinta ed.). México: McGRAW-HILL.

CONAGUA. (2015). Manual de Agua Potable, Alcantarillado y Saneamiento: Modelación Hidráulica y de Calidad del Agua en Redes de Distribución. México.

INAA. (1999). NTON 09-001-99: Normas de Diseño de Abastecimiento de Agua en el Medio Rural. Managua.

Lanza, N. (2012). Texto de ejercicios resueltos de hidráulica 2. Managua.

Loáisiga, H. (2010). Material de Clase: Ingeniería Sanitaria I. Estelí.

Mott, R. (2006). Mecánica de Fluidos. México: PEARSON EDUCATION.

Navarro, S. (2013). Orientaciones de Trabajo de Fin de Curso. Metodología de Investigación. Estelí.

Rossman, L. (1997). Manual de usuario EPANET versión 2.0 vE. Traducción Grupo Multidisciplinar de Modelación de Fluidos Universidad Politécnica de Valencia. Cincinnati.

Saldarriaga, J. (2007). Hidráulica de tuberías. Abastecimiento de agua, redes, riego. Bogotá: Alfaomega.

UCV. (2003). Ingeniería Hidráulica en los Abastecimientos de Agua. Valencia. 

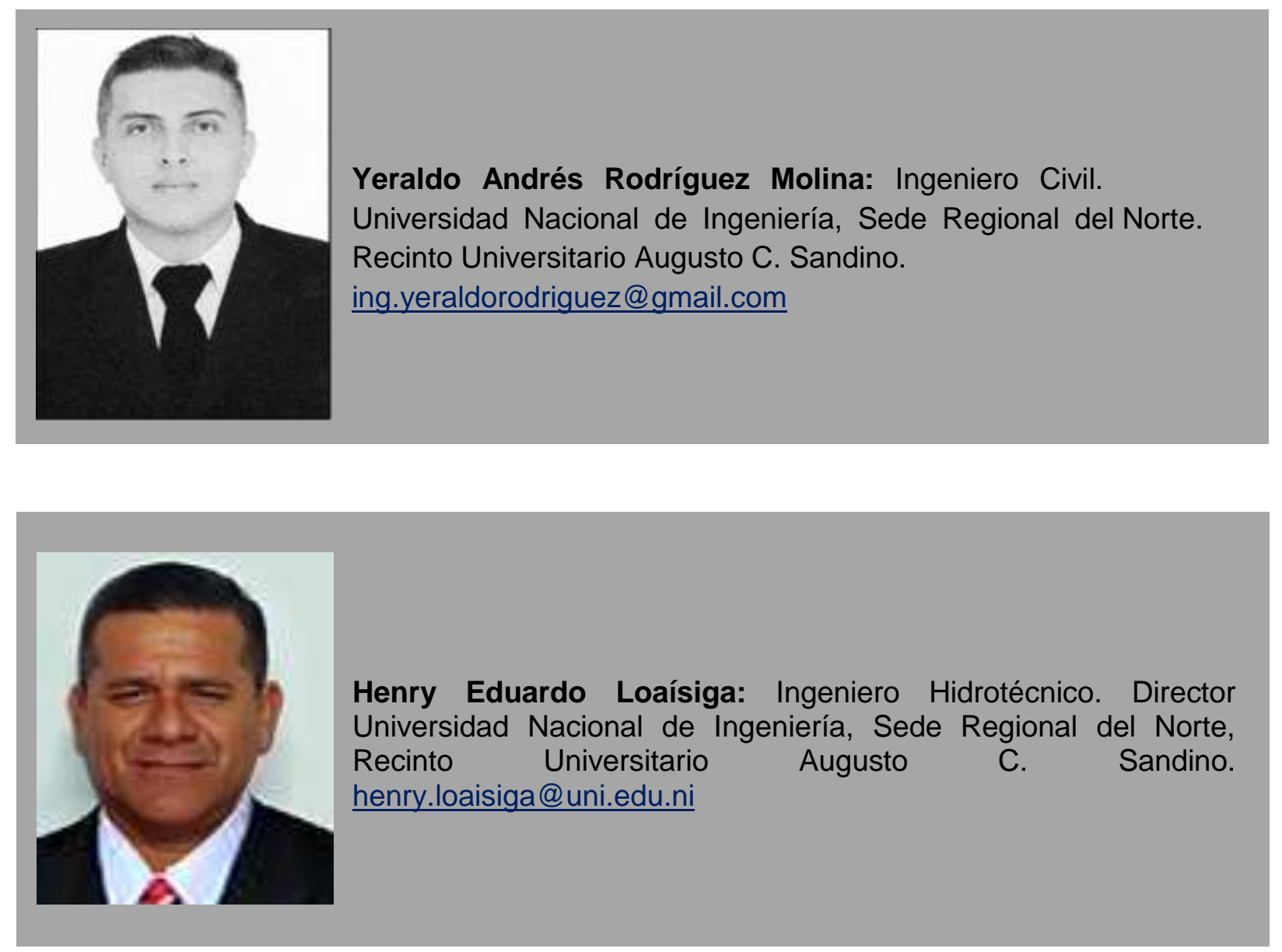